\title{
EFEITOS DA INCLUSÃO OU NÃO DA COVARIÂNCIA GENÉTICA DIRETA-MATERNAL NO MODELO E DOS VALORES REAIS DAS (CO)VARIÂNCIAS SOBRE SUAS ESTIMATIVAS PARA PESO À DESMAMA EM BOVINOS DE CORTE
}

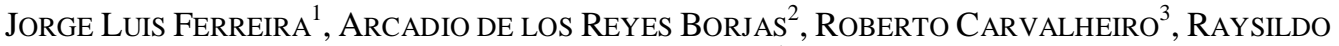 \\ BARBOSA LÔBO ${ }^{4}$ \\ 1 - Professor Doutor Universidade Federal do Tocantins. Araguaína, TO - jorgeuft@gmail.com \\ 2 - Professor Titular do Departamento de Produção Animal, da Escola de Veterinária e Zootecnia da Universidade Federal de Goiás. \\ Goiânia, GO. \\ 3 - Pós-doutor Gensys Cosultoria. Porto Alegre, Rio Grande do Sul, \\ 4 - Professor Associado da FMRP-USP. Diretor-Presidente da ANCP, Ribeirão Preto, São Paulo
}

\section{RESUMO}

Objetivou-se estimar e comparar componentes de (co)variâncias para peso a desmama em bovinos, considerando-se os efeitos da correlação genética diretamaternal $(-0,50 ;-0,25 ;+0,25 ;+0,50)$, da razão entre as variâncias genéticas direta e maternal (75:75; 50:100; 100:50), e da inclusão (M2) ou não (M1) no modelo da covariância genética direta-maternal. Foi realizada simulação estocástica de 20 réplicas de um rebanho fechado, em acasalamento aleatório, durante 20 anos de seleção, com animais base não aparentados, não selecionados e amostrados aleatoriamente. As estimativas de (co)variâncias foram obtidas sob modelo animal unicarácter, usando o aplicativo MTDFREML. Os cenários simulados foram constituídos pelas combinações dos níveis dos três efeitos estudados. O modelo influenciou significativamente $(\mathrm{p}<0,05)$ as estimativas de variâncias genéticas direta, maternal e residual, não sendo significativo sobre a variância de ambiente permanente maternal. O valor da correlação genética direta-maternal influenciou significativamente $(\mathrm{p}<0,05)$ todas as (co)variâncias, e a razão de variâncias exerceu efeito significativo $(\mathrm{p}<0,05)$ apenas sobre as estimativas de variâncias genéticas direta e maternal. Quando a correlação genética direta-maternal foi negativa o M1 subestimou as variâncias direta e maternal, e quando positiva superestimou as mesmas. Estimativas confiáveis e acuradas das (co)variâncias para peso a desmama em bovinos de corte são dependentes da adequação do modelo, bem como dos valores reais das (co)variâncias sendo estimadas.

PALAVRAS-CHAVE: bovinos; simulação; correlação genética direta-maternal; estimativas de (co)variâncias.

\author{
EFFECTS OF NON-INCLUSION OF GENETIC DIRECT-MATERNAL COVARIANCE \\ IN THE MODEL AND REAL VALUES OF (CO)VARIANCES ON THEIR ESTIMATES \\ FOR WEANING WEIGHT IN BEEF CATTLE.
}

\section{ABSTRACT}

This study aimed to compare estimates of (co)variances considering the effect of direct maternal genetic correlation $(-0,50 ;-0,25 ;+0,25 ;+0,50)$; the ratios between the direct and maternal genetic variances (75:75; 50:100; 100:50) and two models which included (M2) or not (M1) the direct maternal genetic covariance. Stochastic simulation of twenty replicates of a closed and randomly mated herd of cattle was carried out for twenty years of selection. The base population was not related, nonselected and randomly sampled. (Co)variance estimates were obtained under single trait animal model using MTDFREML application. The model was not significant 
on estimates of maternal permanent environmental variance. The genetic direct-maternal correlation influenced all (co)variance estimates significantly $(\mathrm{P}<0,05)$. The ratios of genetic direct and maternal variances significantly affected $(\mathrm{P}<0,05)$ only estimates of genetic direct and maternal variances. Regarding negative direct-maternal genetic correlation, M1 underestimated direct and maternal variances while overestimated both when that correlation was positive. Reliable and accurate (co)variance estimates for weaning weight in beef cattle depend on the adequacy of the model as well as their actual values.

KEYWORDS: cattle; direct-maternal genetic correlation; co-variance estimates; simulation.

\section{INTRODUÇÃO}

A eficiência da avaliação genética para características sob efeitos maternais em bovinos (de corte) requer estimativas confiáveis das (co)variâncias correspondentes. A confiabilidade dessas estimativas depende da natureza dos dados disponíveis, do número de registros por vaca, do modelo estatístico e do método de estimação aplicados, da consideração dos efeitos maternais (genéticos e ambientais), do número de mães e avós maternas com registros e do número de gerações presentes nos dados analisados (GERSTMAYR, 1992; ROEHE \& KENNEDY, 1993; MANIATIS \& POLLOT, 2003; HEYDARPOUR et al., 2008; ZAMANI \& MOHAMMADI, 2008).

Quando os efeitos maternais são ignorados há superestimativa da herdabilidade direta $(\mathrm{KOCH}$, 1972), o que pode conduzir a conclusões errôneas, porque toda a variância encontrada, excetuando-se a residual, terá origem atribuída ao efeito genético direto.

MEYER (1992) estimou (co)variâncias para pesos à desmama, em bovinos Hereford, Angus e Zebu cruzado na Austrália, através de seis diferentes modelos, verificando que, quando os efeitos maternais foram ignorados, a herdabilidade direta foi inflada substancialmente.

DIOP et al. (1999) testaram a inclusão dos efeitos de avós no modelo animal com efeitos maternos em animais da raça Gobra, no Senegal, e sugeriram que o valor negativo da correlação genética entre os efeitos direto e materno podia estar relacionado com as condições ambientais em que os animais foram manejados.

Assim, diante das incertezas, tem sido proposto considerar como igual a zero (SCHAEFFER, 2010) a correlação genética entre os efeitos direto e maternal. No entanto, os modelos que a consideram, revelaram serem os mais indicados.

Dessa forma, objetivou-se estimar componentes de (co)variâncias para peso à desmama em bovinos de corte, comparando-se modelos que incluíram ou não a covariância genética direta maternal, sobre dados simulados.

\section{MATERIAL E MÉTODOS}

Foi realizada simulação estocástica de 20 réplicas de um rebanho fechado, em acasalamento aleatório, de 1.000 vacas e 25 touros (população base), não aparentados, não selecionados e amostrados e distribuídos aleatoriamente em 40 grupos de contemporâneos. Os dados foram simulados considerando-se um horizonte de 20 anos de seleção, sob acasalamento aleatório, aproximando-se de condições reais de campo, a exemplo dos grandes rebanhos zebuínos de corte existentes no Brasil. O rebanho foi composto por 1.000 vacas com idades de 3 a 15 anos, acasaladas aleatoriamente, gerando um bezerro por ano. Realizou-se descarte anual das vacas com idade máxima de 15 anos e diagnóstico negativo de gestação. Para cada idade reprodutiva predefiniu-se um índice de concepção (IC) (Tabela 1). Os machos foram selecionados com base em seu valor genético individual. A seleção contemplou reposição anual de $32 \%$ dos touros e $28 \%$ das vacas.

Nos anos seguintes, o critério de seleção de machos e fêmeas também se baseou no valor genético individual. A reposição foi efetuada com animais nascidos três anos antes no próprio rebanho, produzindo sete gerações sobrepostas ao longo dos 20 anos. Dessa forma, houve nascimento anual de 724 bezerros, distribuídos aleatoriamente em 40 grupos contemporâneos (GC), 20 constituídos por machos e os demais por fêmeas. Para garantir a conexidade genética entre os GC foram impostas restrições sobre os números mínimos de filhos por touro (5), bezerros por GC (5), touros por GC (2), conforme proposto por SCHAEFFER (2010).

Como característica sob efeito maternal, considerou-se o peso à desmama (PD), cujo valor para os machos foi acrescido em $15 \mathrm{~kg}$, em relação às fêmeas. Neste caso, a simulação dos efeitos genéticos direto e maternal foi gerada 
simultaneamente, já que tais efeitos são geneticamente correlacionados (SCHAEFFER, 2010). Esse processo sustenta-se na decomposição de Cholesky da matriz G de (co)variâncias genéticas, a qual pode ser expressa pelo produto de uma matriz vezes sua transposta, isto é:

$$
\boldsymbol{G}=\left(\begin{array}{cc}
\sigma_{\boldsymbol{a}}^{2} & \sigma_{\boldsymbol{a m}} \\
\sigma_{\boldsymbol{a} \boldsymbol{m}} & \sigma_{\boldsymbol{m}}^{2}
\end{array}\right)=\left(\begin{array}{cc}
\boldsymbol{C}_{11} & 0 \\
\boldsymbol{C}_{12} & \boldsymbol{C}_{22}
\end{array}\right)\left(\begin{array}{cc}
\boldsymbol{C}_{11} & \boldsymbol{C}_{12} \\
0 & \boldsymbol{C}_{22}
\end{array}\right)
$$

em que $C$ é uma matriz triangular inferior com os valores $C_{11}, C_{12}$ e $C_{22}$ correspondentes às componentes $\sigma_{\text {a }}^{2}, \sigma_{\text {am }}^{2}$ e $\sigma_{m}^{2}$, respectivamente.

Para o processo de simulação foram desenvolvidos diversos programas utilizando o SAS (SAS, 2002). O PD foi simulado segundo o seguinte modelo: $\mathrm{PD}=$ $b_{0}+b_{1} * i d v+b_{2} * i d v^{2}+e g c+v g a+v g m v+a p+e$, em que; $\mathrm{PD}=$ peso ao desmame; $b_{0}=$ intercepto (136 kg.); $\left(b_{1}=6,551 ; b_{2}=-0,417\right)$ coeficientes de regressão parciais linear e quadrático, respectivamente; $i d v=$ idade da vaca ao parto em anos; $e g c=$ efeito do grupo contemporâneo; $v g a=$ valor genético aditivo direto do animal; vgmv = valor genético aditivo maternal da vaca (mãe); $a p=$ efeito ambiental permanente maternal; $e=$ efeito residual.
Tabela 1. Número de vacas expostas à reprodução e índice de concepção (IC) segundo idade (anos) no processo de simulação

\begin{tabular}{lll}
\hline Idade & Vacas expostas & Vacas paridas (IC \%) \\
\hline 3 & 280 & $224(80,00)$ \\
4 & 224 & $112(50,00)$ \\
5 & 112 & $89(79,46)$ \\
6 & 89 & $71(70,78)$ \\
7 & 71 & $56(78,87)$ \\
8 & 56 & $44(78,57)$ \\
9 & 44 & $35(79,55)$ \\
10 & 35 & $28(80,00)$ \\
11 & 28 & $22(78,57)$ \\
12 & 22 & $17(77,27)$ \\
13 & 17 & $13(76,47)$ \\
14 & 13 & $09(69,23)$ \\
15 & 09 & $04(44,44)$ \\
\hline Total & 1.000 & $724(72,40)$ \\
\hline
\end{tabular}

Os valores egc, ap e $e$ foram amostrados de distribuição Normal com média zero e variâncias $\sigma^{2}$ egc $(e g c \sim \mathrm{N}(0,450)), \quad \sigma_{\text {ap }}(a p \sim \mathrm{N}(0,45))$ е $\sigma^{2}{ }_{\mathrm{e}}$ $(e \sim \mathrm{N}(0,225))$, respectivamente. Nas gerações seguintes à população base, os valores genéticos aditivos direto (vga) e maternal (vgm) de cada indivíduo foram calculados conforme as equações a seguir:

$$
\begin{gathered}
\boldsymbol{v g a}=1 / 2\left(\boldsymbol{v g a}_{t}+\boldsymbol{v g a}_{v}\right)+A M_{a} \quad \boldsymbol{v g m}=1 / 2\left(\boldsymbol{v g m}_{t}+\operatorname{vgm}_{v}\right)+A M_{m} \\
A M_{a}=\left(z_{1} C_{11}\right) \sqrt{0,5\left(1-\left(F_{t}+F_{v}\right) / 2\right)} \quad A M_{m}=\left(z_{1} C_{12}+z_{2} C_{22}\right) \sqrt{0,5\left(1-\left(F_{t}+F_{v}\right) / 2\right)}
\end{gathered}
$$

em que: $v g a=$ valor genético aditivo direto do bezerro; $v g m=$ valor genético aditivo maternal do bezerro; $v g a_{t}=$ valor genético aditivo direto do touro; $v g a_{v}=$ valor genético aditivo direto da vaca; $v g m_{t}=$ valor genético aditivo maternal do touro; $v \mathrm{gm}_{v}=$ valor genético aditivo maternal da vaca; $A M_{a}, A M_{m}=$ contributos da amostragem Mendeliana aleatória aos valores genéticos aditivos direto e maternal, respectivamente; $z_{1}, z_{2}=$ desvios normais aleatórios; $C_{11}, C_{12}, C_{22}=$ termos da decomposição de Cholesky da matriz $G$, correspondentes às componentes $\sigma^{2}$ a, $\sigma^{2}$ am e $\sigma^{2}$ m, respectivamente; $F_{t}$ e $F_{v}=$ coeficientes de consanguinidade do touro (pai) e da vaca (mãe), respectivamente. A partir do quarto ano aconteceram acasalamentos entre parentes, gerando indivíduos com algum grau de consanguinidade, parte dos quais acasalaram a partir do sétimo ano. Os efeitos da amostragem Mendeliana assumiram-se independentes de $v g a_{t}, v g a_{v}, v g m_{t}$ e $v g m_{v}$ (BULMER,
1971). A decomposição de Cholesky da matriz de (co)variâncias genética $(G)$ foi obtida por meio da função root do PROC IML do SAS (2002).

As estimativas de (co)variâncias e as predições de valores genéticos, foram obtidas pelo método de Máxima Verossimilhança Restrita (REML) através de modelo animal unicaráter, usando o aplicativo MTDFREML (Multiple-Trait Derivative-Free Restricted Maximum Likelihood), desenvolvido por BOLDMAN et al. (1995).

O modelo linear misto para descrever cada observação foi:

$$
Y=X \beta+Z_{1} a+Z_{2} m+Z_{3} a p+e
$$

em que; $\boldsymbol{Y}=$ vetor das observações da característica (PD); $\boldsymbol{X}=$ matriz de incidência dos efeitos fixos; $\boldsymbol{\beta}=$ vetor dos efeitos fixos; $Z_{1}=$ matriz de incidência do efeito genético aditivo direto de cada animal; $\boldsymbol{a}=$ vetor de efeitos aleatórios genéticos aditivos diretos; $Z_{2}=$ matriz de incidência do efeito genético aditivo maternal de cada animal; $\boldsymbol{m}=$ vetor de efeitos 
aleatórios genéticos aditivos maternais; $\boldsymbol{Z}_{3}=$ matriz de incidência do efeito do ambiente permanente maternal; $\boldsymbol{a p}=$ vetor de efeitos aleatórios do ambiente permanente maternal; $\boldsymbol{e}=$ vetor de efeitos aleatórios residuais.

As distribuições e matriz de (co)variâncias dos efeitos aleatórios foram assim definidas:

$$
\begin{gathered}
E[a]=E[m]=E[a p]=E[e]=0, \\
E[y \mid a, m, a p]=X \beta+Z_{1} a+Z_{2} m+Z_{3} a p \\
{\left[\begin{array}{l}
\boldsymbol{a} \\
\boldsymbol{m} \\
\boldsymbol{p} \\
\boldsymbol{e}
\end{array}\right]=\left[\begin{array}{cccc}
\boldsymbol{A} \sigma_{\boldsymbol{a}}^{2} & \boldsymbol{A} \sigma_{\boldsymbol{a m}} & 0 & 0 \\
\boldsymbol{A} \sigma_{\boldsymbol{a m}} & \boldsymbol{A} \sigma_{\boldsymbol{m}}^{2} & 0 & 0 \\
0 & 0 & \boldsymbol{I}_{\boldsymbol{v}} \sigma_{a p}^{2} & 0 \\
0 & 0 & 0 & \boldsymbol{I}_{\boldsymbol{N}} \sigma_{\boldsymbol{e}}^{2}
\end{array}\right]}
\end{gathered}
$$

em que: $\sigma^{2}{ }_{a}=$ variância genética aditiva direta; $\sigma^{2} \mathrm{~m}$ $=$ variância genética aditiva maternal; $\sigma_{\mathrm{am}}=$ covariância genética entre os efeitos aditivos direto e maternal; $A$ = matriz de parentesco; $\sigma_{\text {ap }}^{2}=$ variância do ambiente permanente maternal; $\sigma^{2}{ }_{e}=$ variância residual; $\mathrm{I}_{\mathrm{v}}, \mathrm{I}_{\mathrm{N}}=$ matrizes identidade de ordens; $\mathrm{v}=$ número de vacas (mães dos animais com dados) e $\mathrm{N}$ = número total de animais com dados.

Dois modelos de análises foram usados para estimar os componentes de (co)variâncias: $\mathrm{M}_{1}$ - modelo animal que considerou a covariância genética entre os efeitos direto e maternal igual a zero $(\operatorname{cov}=0)$; e $\mathrm{M}_{2}$ em que o valor da covariância genética diretamaternal foi considerado conforme a simulação dos dados.

O modelo linear misto para descrever cada observação, foi:

$$
Y=X \beta+Z_{1} a+Z_{2} m+Z_{3} a p+e
$$

em que: $\boldsymbol{Y}=$ vetor das observações da característica (PD); $\boldsymbol{X}=$ matriz de incidência dos efeitos fixos; $\boldsymbol{\beta}=$ vetor dos efeitos fixos; $\boldsymbol{Z}_{\boldsymbol{1}}=$ matriz de incidência do efeito genético aditivo direto de cada animal; $\boldsymbol{a}=$ vetor de efeitos aleatórios genéticos aditivos diretos; $\boldsymbol{Z}_{2}=$ matriz de incidência do efeito genético aditivo maternal de cada animal; $\boldsymbol{m}=$ vetor de efeitos aleatórios genéticos aditivos maternais; $\boldsymbol{Z}_{3}=$ matriz de incidência do efeito do ambiente permanente maternal; $\boldsymbol{a p}=$ vetor de efeitos aleatórios do ambiente permanente maternal; $\boldsymbol{e}=$ vetor de efeitos aleatórios residuais.

Os cenários foram constituídos considerando-se quatro valores de correlação genética $(-0,50 ;-0,25$; $+0,25$ e +0,50), três razões entre as variâncias genéticas direta e maternal $(75: 75 ; 50: 100 ; 100: 50)$ e dois modelos $\left(\mathrm{M}_{1}\right.$ e $\left.\mathrm{M}_{2}\right)$. Dessa forma, foram constituídos 24 cenários (4 (C) x 3 (R) x 2 (M)) = 24 cenários).

As estimativas de (co)variâncias obtidas em cada cenário, foram submetidas à analise de variância pelo procedimento GLM (General Linear Model) pelo sistema de analises estatísticas (SAS, 2002), conforme o modelo:

$$
\begin{gathered}
\mathrm{Y}_{\mathrm{ijkl}}=\mu+\mathrm{C}_{\mathrm{i}}+\mathrm{R}_{\mathrm{j}}+\mathrm{M}_{\mathrm{k}}+\text { (Interações Duplas) }+ \\
\text { (Interação Tripla) }+\mathrm{e}_{\mathrm{ijkl}}
\end{gathered}
$$

em que: $\mathrm{Y}_{\mathrm{ijkl}}=$ estimativas de (co)variâncias (i.e., $\sigma_{\mathrm{a}}^{2}$, $\sigma^{2}{ }_{m}, \sigma_{p}^{2}, \sigma_{e}^{2}$ ); $\mu=$ média geral; $C_{i}=$ efeito do i-ésimo valor da correlação genética direta-maternal (-0,50; 0,$25 ;+0,25$ e +0,50); $R_{j}=$ efeito da j-ésima razão entre variâncias genéticas direta e maternal (75:75; 50:100; 100:50); $\mathrm{M}_{\mathrm{k}}=$ efeito do k-ésimo modelo ( $\mathrm{M}_{1}$ e $M_{2}$ ); Interações Duplas $=C_{i} \times R_{j} ; C_{i} \times M_{k} ; R_{j} \times M_{k}$; Interação Tripla $=C_{i} \times R_{j} \times M_{k}$; $e_{i j k l}=$ efeito residual aleatório.

$\mathrm{O}$ erro padrão quadrático $(\mathrm{EP})^{2}$ da média das 20 réplicas para cada cenário foi usado como fator de ponderação nas análises. A significância de cada efeito sobre as estimativas de (co)variâncias foi testada pelo procedimento GLM do SAS (2002). O erro padrão (EP) das estimativas de cada cenário foi utilizado como critério de precisão e o viés médio, como critério de acurácia.

\section{RESULTADOS E DISCUSSÃO}

Os resultados das análises de variâncias para peso a desmama sobre as (co)variâncias estimadas estão apresentados na Tabela 2. As estimativas de (co)variâncias para a característica sob efeito maternal foi significativamente influenciada $(p<0,0001)$ pela correlação genética direta-maternal (C), apresentando interações significativas com a razão de variâncias (R) e o modelo (M).

A interação $C x \mathrm{R}$ não foi significativa para as estimativas de variância genética maternal, enquanto que as estimativas da variância de ambiente permanente maternal não foram significativamente influenciadas pela interação $C x M$. A interação $C x R$ teve contribuição importante ao ajuste do modelo, para as estimativas da variância de ambiente permanente maternal (8\%), sendo reduzida $(0,7 \%$ e $1 \%)$ para as demais estimativas (i.e., $\sigma^{2}{ }_{a}, \sigma^{2}$ e).

A interação $C x M$ teve efeito significativo $(p<0,0001)$ sobre as estimativas da variância genética maternal, variância genética direta e variância residual, contribuindo ao ajuste do modelo com $9 \%, 11 \%$ e $26 \%$, respectivamente.

$\mathrm{O}$ efeito do modelo não foi significativo sobre as estimativas de variância genética maternal e variância de ambiente permanente maternal. Os resultados no modelo que não considerou a covariância genética direta-maternal $\left(\mathrm{M}_{1}\right)$, para as 
estimativas de variâncias genéticas direta e maternal, mostraram um viés negativo de (-31,36\% e -15,62\%; $-36,61$ e $-13,23)$ respectivamente, quando a correlação genética direta-maternal foi negativa ($0,50$ e - 0,25$)$.

Tabela 2. Significâncias dos efeitos sobre as estimativas de (co)variâncias para a característica PD em bovinos de corte

\begin{tabular}{l|lllll}
\hline Efeitos & $G l$ & $\sigma_{\boldsymbol{a}}^{2}$ & $\sigma_{\boldsymbol{m}}^{2}$ & $\sigma_{\boldsymbol{a p}}^{2}$ & $\sigma_{\boldsymbol{e}}^{2}$ \\
\hline$C$ & 3 & $* * *$ & $* * *$ & $* * *$ & $* * *$ \\
$R$ & 2 & $* * *$ & $* * *$ & $n s$ & $n s$ \\
$M$ & 1 & $*$ & $n s$ & $n s$ & $*$ \\
$C \times R$ & 6 & $* *$ & $n s$ & $* * *$ & $* *$ \\
$C \times M$ & 3 & $* * *$ & $* * *$ & $n s$ & $* * *$ \\
$R \times M$ & 2 & $n s$ & $n s$ & $n s$ & $n s$ \\
$C \times R \times M$ & 6 & $n s$ & $n s$ & $n s$ & $n s$ \\
\hline$R^{2}$ & & 0,816 & 0,846 & 0,173 & 0,611 \\
\hline
\end{tabular}

Gl =Graus de liberdade; $M=$ Modelo $\left(M_{1}\right.$ e $\left.M_{2}\right)$; $C=$ correlação genética direta-maternal; $\mathrm{R}=$ razão entre variâncias genéticas direta e maternal; $\mathrm{MxC}$ =interação modelo x correlação; $\mathrm{MxR}=$ interação modelo x razão; C x R=interação correlação x razão; M x C x R= interação modelo x correlação x razão; * =p $<0,05$; $* *=\mathrm{p}<0,001 ; * * *=\mathrm{p}<0,0001 ;$ ns $=$ não significativo; Coeficiente de determinação do modelo, $\mathrm{R}^{2}=$ (SQmodelo / SQtotal). $\sigma^{2}=$ variância genética aditiva direta; $\sigma^{2}{ }_{m}=$ variância genética aditiva maternal; $\sigma^{2}$ ap $=$ variância do efeito de ambiente permanente maternal; $\sigma^{2}{ }_{\mathrm{e}}=$ variância atribuída ao efeito ambiental.

Entretanto, quando a correlação genética direta-maternal foi positiva $(+0,25$ e $+0,50)$, no mesmo modelo, houve aumento na magnitude das estimativas de variâncias genéticas dos efeitos direto e maternal, sendo as mesmas superestimadas.

No modelo que incluiu a covariância genética direta-maternal $\left(\mathrm{M}_{2}\right)$, obteve-se o menor viés das estimativas de variâncias genéticas direta e maternal, com magnitudes de $(3,3$ e 1,0$)$ e $(1,38$ e 3,69$) \mathrm{kg}^{2}$, quando $\mathrm{C}=-0,50$ e $-0,25$, respectivamente, e $(0,99$ e $4,35)$ e $(0,71$ e 3,57$) \mathrm{kg}^{2}$, quando $\mathrm{C}=+0,25$ e $+0,50$, respectivamente.

Estes resultados concordam com os reportados por HEYDARPOUR et al. (2008) em estudo de simulação. E de forma similar, aos reportados por CLÉMENT et al. (2001) que observaram que a confiabilidade das estimativas de (co)variâncias foi dependente do valor da correlação genética real nos dados.

Quando a correlação genética direta-maternal é considerada igual a zero $\left(\mathrm{M}_{1}\right)$, os valores das estimativas de variância genética direta e maternal são sempre viesados.

Assim, verificou-se que quando a correlação genética direta-maternal foi negativa $(-0,50$ e $-0,25)$ as estimativas de variância genética direta e maternal foram subestimadas, chegando a 1,32 vezes menor que o valor real. E quando a correlação genética direta-maternal foi positiva $(+0,25$ e $+0,50)$ as estimativas de variâncias genética direta e maternal foram superestimadas, chegando a 0,77 vezes maior que o valor real. Corroborando os resultados encontrados por CLÉMENT et al. (2001) que demonstraram que quando a correlação genética direta-maternal é considerada nula, os valores das estimativas de variância genética direta e da herdabilidade direta são superestimados, chegando às vezes, esse valor, a duplicar em relação aos valores verdadeiros.

As interações $R x M$ e $C x R x M$ não exerceram efeitos significativos sobre as estimativas de (co)variâncias para peso a desmama em bovinos de corte.

Dessa forma, assumindo que as condições aqui simuladas sejam similares às dos dados reais, revelase que a utilização de modelos que não incluem a covariância genética direta-maternal determinam estimativas não confiáveis, comprometendo as estimações das (co)variâncias, dos parâmetros genéticos e da ordem de classificação dos animais pelos seus valores genéticos preditos.

Observou-se no $M_{2}$ que, o viés médio nos diferentes cenários, das estimativas de variâncias genéticas direta e maternal e variância residual foram de $+2,21 ;+2,19$ e $-0,02$, respectivamente. Sob o modelo $\left(\mathrm{M}_{2}\right)$, as estimativas mais acuradas e precisas, para as variâncias genéticas direta e maternal e a variância residual, foram obtidas quando $\mathrm{C}=+0,25$ e a razão 1:1 entre variâncias genéticas direta e maternal.

Na Figura 1 são apresentadas as magnitudes do viés das estimativas de variâncias (\%) e da covariância direta-maternal (valor real), segundo valores reais da razão entre as variâncias genéticas direta e maternal e da correlação genética diretamaternal, sob modelos incluindo $\left(\mathrm{M}_{2}\right)$ ou não $\left(\mathrm{M}_{1}\right)$ a covariância genética direta-maternal.

Na Figura 1 observa-se que sob o modelo $\mathrm{M}_{1}$, quando a correlação genética direta-maternal (C) foi negativa, as variâncias genéticas direta e maternal foram subestimadas, enquanto a variância residual foi viesada positivamente. Opostamente, quando C foi positiva, as $\sigma^{2}$ a $\quad \sigma^{2}{ }_{m}$ foram viesadas positivamente, com subestimação da $\sigma^{2}$ e.

Resultados similares foram reportados por CLÉMENT et al. (2001) que observaram que a confiabilidade das estimativas de (co)variâncias foram dependentes do valor real da correlação genética direta-maternal nos dados. 


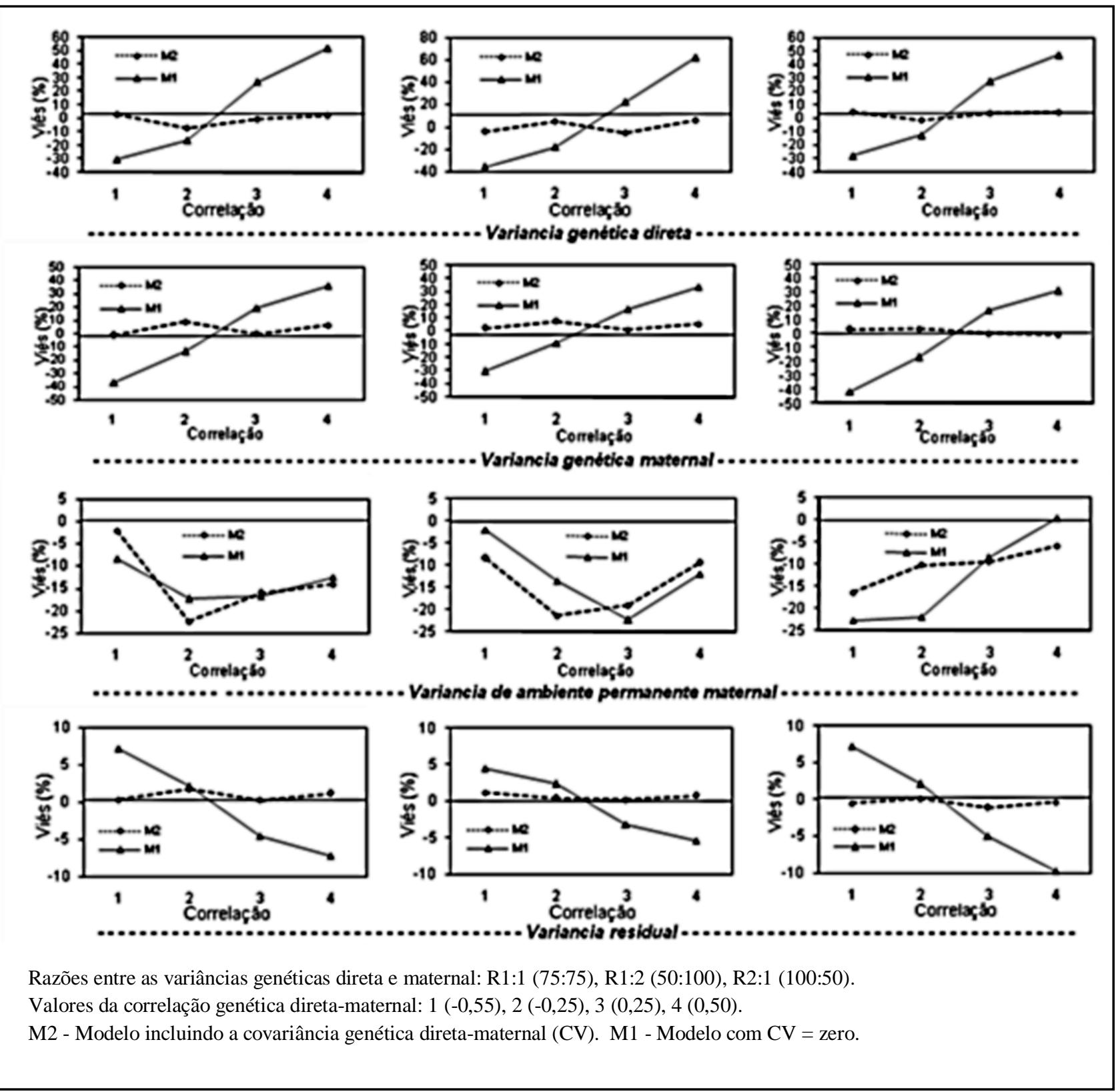

Figura 1. Magnitudes do viés das estimativas de variâncias (\%) segundo valores reais das razões entre as variâncias genéticas direta e maternal e da correlação genética direta-maternal, sob modelos incluindo (M2) ou não (M1) a covariância genética direta-maternal.

Diversos autores (ELER et al., 1989; MEYER, 1992; FERNANDES \& FERREIRA, 2000; UTRERA, 2008) têm estimado correlações genéticas de valor negativo entre os efeitos direto e maternal, com dados de campo. Este antagonismo genético tem sido levado em conta nas avaliações genéticas de muitas raças de bovinos de corte, com valores, em média, -0,29 para Brangus, -0,21 para Gelbvieh, 0,29 para Beefmaster, -0,18 para Senepol e -0,09 para o Shortorn (BIF, 2010). Este mesmo guia recomenda também nas avaliações genéticas de algumas raças, valor zero para a correlação genética entre os efeitos direto e maternal, como Angus,
Braford, Hereford, Limousin, Red Angus, Romagnola e Santa Gertrudis.

Na realidade, essas recomendações se baseiam principalmente na dificuldade da separação dos diferentes efeitos e suas correlações sobre o desempenho fenotípico, presente em dados de campo. SCHAEFFER (1984) discutiu os efeitos de valores de correlação incorretos em análises multivariadas, concluindo que o PEV aumentou com o uso incorreto de covariâncias. Da mesma forma, HENDERSON (1975) comentou que o uso de variâncias e matriz de covariâncias incorretas no modelo misto faz aumentar as variâncias dos erros de 
predição (PEV).

Nos gráficos da Figura 1, observa-se que ao se considerar a covariância genética direta-maternal igual a zero, em todos os cenários analisados, as estimativas de (co)variâncias são todas viesadas. No entanto, quando a covariância foi considerada diferente de zero, os vieses foram menores, e estimativas de variâncias genéticas direta e maternal mais confiáveis foram obtidas quando a variância direta foi maior que a maternal.

Assim, a confiabilidade e precisão das estimativas de (co)variâncias e consequentemente dos parâmetros genéticos é conseguida com modelos que incluem a covariância genética direta-maternal.

As estimativas da variância de ambiente permanente maternal foram todas viesadas negativamente, e deforma similar em magnitude sob ambos os modelos $\left(\mathrm{M}_{1}\right.$ e $\left.\mathrm{M}_{2}\right)$ e para qualquer um dos cenários. Essa situação é extremamente particular e curiosa, uma vez que no foi encontrado na literatura consultada nenhum resultado referido ao viés da estimativa dessa variância.

A confiabilidade das estimativas de variância residual foi maior no modelo que incluiu a covariância genética direta-maternal (M2), indicando, mais uma vez, que a inclusão da covariância genética direta-maternal no modelo é de extrema importância.

A maioria dos resultados da literatura, para as estimativas das (co)variâncias de que trata o presente trabalho, mostram correlação negativa, de média a alta entre os efeitos genéticos aditivos direto e maternal, e estimativas superiores da variância genética direta relativo à maternal. Como exemplo, UTRERA (2008) encontraram para peso a desmama, estimativas médias de herdabilidade direta, maternal e correlação genética entre os efeitos direto e maternal, da ordem de $0,27,0,17$ e $-0,23$, respectivamente.

Assim, assumindo as condições simuladas semelhantes daquelas dos dados reais, fica claro que o modelo que inclui a covariância genética diretamaternal permite as estimativas mais confiáveis das (co)variâncias, exceto a variância de ambiente permanente maternal, o qual se ilustra no lado esquerdo dos gráficos da Figura 1 (correlações reais 1 e 2) para a razão de variâncias direta/maternal 2:1.

\section{CONCLUSÕES}

A não inclusão da covariância genética diretamaternal no modelo determina estimativas viesadas das covariâncias para caracteres sob efeito maternal, gerando pouca confiabilidade das mesmas, assim como dos parâmetros genéticos derivados, gerando, consequentemente, predições menos acuradas dos valores genéticos.

A recomendação de vários autores de não considerar a covariância genética direta-maternal no modelo de análise não parece uma boa estratégia para estimação de (co)variâncias e parâmetros genéticos do crescimento até a desmama, pois verificou-se que isto leva a estimativas viesadas das (co)variâncias, interferindo nas estimativas dos parâmetros genéticos, na predição dos valores genéticos dos animais e no ganho genético por seleção.

\section{REFERENCIAS}

BIF. Guidelines for uniform beef improvement programs $\left(9^{\text {th }}\right.$ Ed.), Beef Improvement Federation, USA. $2010 . \quad$ Disponível em: http://www.beefimprovement.org/PDFs/guidelines/2010\% 20Guidelines\%20,\%209th\%20Edition.pdf. Acesso em 03/06/2011.

BOLDMAN, K.G., KRIESE, L.A., VAN VLECK, L.D., VAN TASSELL, C.P., KACHMAN, S.D. A manual for use of MTDFREML: a set of programs to obtain estimates of variances and covariances [DRAFT]. Lincoln: Department of Agriculture/Agriculture Research Service, 1995.115p.

BULMER, M.G., The effect of selection on genetic variability. The American Naturalist, v.105, p.201-211, 1971.

CLÉMENT, V., BIBÉ, B., VERRIER, E., ELSEN, J.M., MANFREDI, E., BOUX, J., HANOCQ, E. Simulation analysis to test the influence of model adequacy and data structure of genetic parameters for traits with direct and maternal effects. Genetics Selection Evolution, v.33, 369-395, 2001.

DIOP, M., DODENHOFF, J., Van VLECK, L. D. Estimates of direct, maternal and grandmaternal genetic effects for growth traits in Gobra cattle. Genetics and Molecular Biology, v. 22, n. 3, p. 363-367,1999.

ELER, J.P., LÔBO, R.B., DUARTE, F.A.M. Avaliação dos efeitos genéticos direto e materno em pesos de bovinos da raça Nelore criados no Estado de São Paulo. Revista Brasileira de Zootecnia, v.18, n.2, p.112-123, 1989.

FERNANDES, H.D., FERREIRA, G.B. Estudo comparativo de sete diferentes modelos estatísticos para a característica ganho de peso em bovinos de corte. Revista Brasileira de Zootecnia, v.29, n.5, p.1340-1348, 2000. 
GERSTMAYR, S. Impact of the data structure on the reliability of the estimated genetic parameters in an animal model with maternal effects, Journal of Animal Breeding Genetics v.109, p.321-326, 1992.

HENDERSON, C.R. Comparison of alternative sire evaluation methods. Journal of Animal Science, v.41, p.760-770, 1975.

HEYDARPOUR, M., SCHAEFFER, L., YAZDI, H. Influence of population structure on estimates of direct and maternal parameters. Journal of Animal Breeding Genetics, v.125, p.89-99, 2008.

$\mathrm{KOCH}$, R.M. The role of maternal effects in animal breeding: VI. Maternal effects in beef cattle. Journal of Animal Science, v.35, p.1316-1323, 1972.

MANIATIS, N., POLLOT, G.E. The impact of data structure on genetic (co)variance components of early growth in sheep, estimated using an animal model with maternal effects. Journal of Animal Science, v.81, p.101108, 2003.

MEYER, K. Bias and sampling covariances of estimates of variance components due to maternal effects. Genetics Selection Evolution., v. 24, p.487-509, 1992.
ROEHE, R., KENNEDY, B.W. The influence of maternal effects on accuracy of evaluation of litter size in swine. Journal of Animal Science, v.71, p.2353-2364, 1993.

SCHAEFFER, L. R. Sire and cow evaluation under multiple trait models. Journal of Dairy Science, v. 67, p.1567-1580, 1984.

SCHAEFFER, L.R. Linear Models and Animal Breeding. Centre for Genetic Improvement of Livestock. Department of Animal and Poultry Science, 2010. European course. 314 p. Disponível em: http://www.aps.uoguelph.ca/ /rs/ABModels/DATA/Euro peNotes.pdf. Acesso em 20/07/2011.

STATISTICAL ANALYSIS SYSTEMS - SAS. User's guide. Version 8.2. 1 ed. Cary: 956p. 2002.

UTRERA, A. Estimadores de parámetros genéticos para características de crecimiento predestete de bovinos. Revisión. Técnica Pecuaria en México, v. 46, p. 37-67, 2008.

ZAMANI, P., MOHAMMADI, H. Comparison of different models for estimation of genetic parameters of early growth traits in the Mehraban sheep. Journal of Animal Breeding and Genetics, v.125, n.1, p.29-34, 2008.

Protocolado em: 08 fev. 2009 Aceito em: 27 jul. 2011. 